\title{
Alveolar soft part sarcoma: Clinicopathological analysis and imaging results
}

\author{
PENG-FEI QIAO ${ }^{1}$, LING-HUI SHEN ${ }^{2}$, YANG GAO ${ }^{1}$, YING-CHUN MI ${ }^{3}$ and GUANG-MING NIU ${ }^{1}$ \\ ${ }^{1}$ Department of Magnetic Resonance Imaging, Affiliated Hospital of Inner Mongolia Medical University; \\ ${ }^{2}$ The Third Surgical Ward, Hospital of Inner Mongolia Armed Police Corps, Hohhot, Inner Mongolia 010050; \\ ${ }^{3}$ Department of Radiology, Tuo Ke Tuo Country Hospital of Inner Mongolia, Hohhot, Inner Mongolia 010200, P.R. China
}

Received October 24, 2014; Accepted August 7, 2015

DOI: $10.3892 / \mathrm{ol} .2015 .3694$

\begin{abstract}
Alveolar soft part sarcoma (ASPS) is a rare, malignant, soft-tissue tumor that accounts for $\sim 1.2 \%$ of all soft-tissue sarcomas. Due to its low incidence, clinicians often overlook the diagnosis. However, it is difficult to form an accurate diagnosis prior to surgery due to the lack of experience in imaging diagnosis. The present study reviewed the pathological images, and the computed tomography and magnetic resonance imaging data of 6 ASPS cases in order to investigate the clinicopathological and imaging characteristics of the tumor. The present study indicated that the magnetic resonance imaging (MRI) appearances of ASPS are nonspecific, but malignancy may be determined to a certain degree, which may aid in diagnosis prior to surgery and provides information for treatment guidance.
\end{abstract}

\section{Introduction}

Alveolar soft part sarcoma (ASPS) is a rare, malignant, soft-tissue tumor that accounts for $\sim 1.2 \%$ of all soft-tissue sarcomas (1). ASPS belongs to the group of malignant tumors with uncertain differentiation. With regard to molecular genetics, it is characterized by an unbalanced translocation, $\operatorname{der}(17) \mathrm{t}(\mathrm{X} ; 17)(\mathrm{p} 11 ; \mathrm{q} 25)(2)$. The most common age of onset is between 15 and 35 years old, and onset prior to the age of 5 years old or after 50 years old is rare. It has been reported (3) that the incidence of the tumor in women is higher than that in men, particularly among women under the age of 25 years old. The most common site of tumor occurrence is in the deep soft tissues of the limb, particularly in the thigh, while it may also occur in rarer sites, such as the head and neck, mediastinum, retroperitoneum, breasts and orbits (4). ASPS grows

Correspondence to: Dr Guang-Ming Niu, Department of Magnetic Resonance Imaging, Affiliated Hospital of Inner Mongolia Medical University, 1 Tong Dao Street, Hohhot, Inner Mongolia 010050, P.R. China

E-mail: niugming@gmail.com

Key words: the differential, alveolar soft part sarcoma, imaging and clinicopathological manifestations slowly, with clinical symptoms that are not pronounced, so the tumor is not easily identified (4). ASPS is also prone to early metastases and has a high recurrence rate following conservative surgical excision. Therefore, the prognosis of the tumor is poor. Careful analysis of the imaging and clinicopathological features, as performed in the present study, will be useful for determining an accurate diagnosis.

\section{Materials and methods}

Patients. A total of 2 males and 4 females with ASPS confirmed by surgery and pathology were treated the Affiliated hospital of Inner Mongolia Medical University (Hohhot, China) between October 2013 and June 2014. . The patient ages ranged from 16 to 45 years old, with a mean of 31.4 years old. The locations of the tumor included 1 case in the thigh, 1 case in the leg, 1 case in the upper arm, and 3 cases in the gluteus muscles and iliopsoases. The duration of the disease ranged between 3 months to 3 years, and the main clinical manifestation was an increasingly soft-tissue mass with pain.

Treatment and analysis. The patients underwent computed tomography (CT) scans, and plain and enhanced magnetic resonance imaging prior to surgery. The location, size, morphology and enhanced pattern of the tumors were analyzed and evaluated by two experts separately. Following radical surgical resection, the pathological sections were analyzed using different methods in the paraffin-embedded tissue samples: (i) Hematoxylin-eosin (HE) staining, (ii) periodic acid-schiff (PAS) staining and (iii) immunohistochemical staining (Envision methods). MyoD1 (cytoplasmic staining), desmin, NSA, vimentin, AE1/AE3, cytokeratin, epithelial membrane antigen, smooth muscle actin, muscle-specific actin and synaptophysin were assessed.

\section{Results}

All tumors were located at the deep muscles. The mean size of the tumors was $4.1 \times 4.8 \times 3.3 \mathrm{~cm}$. In total, 5 tumors were lobulated in shape and 3 tumors exhibited peritumoral soft-tissue nodules. On CT, the tumor density was even, with marked enhancement on contrast-enhanced imaging (Fig. 1). On MRI, the signal intensity was homogeneous, with isointensity 


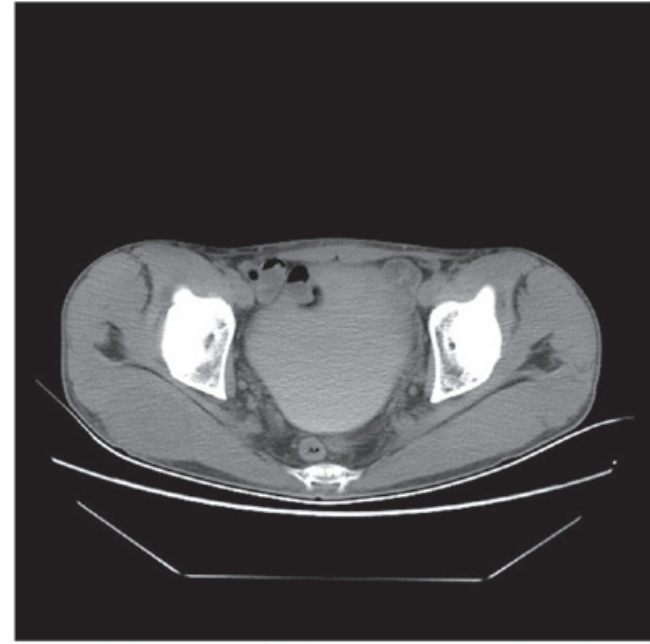

plain scan

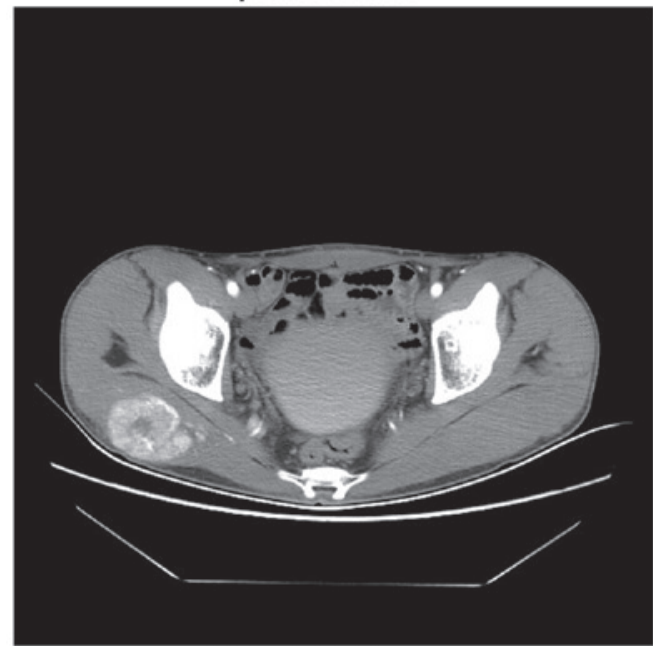

Arterial phase

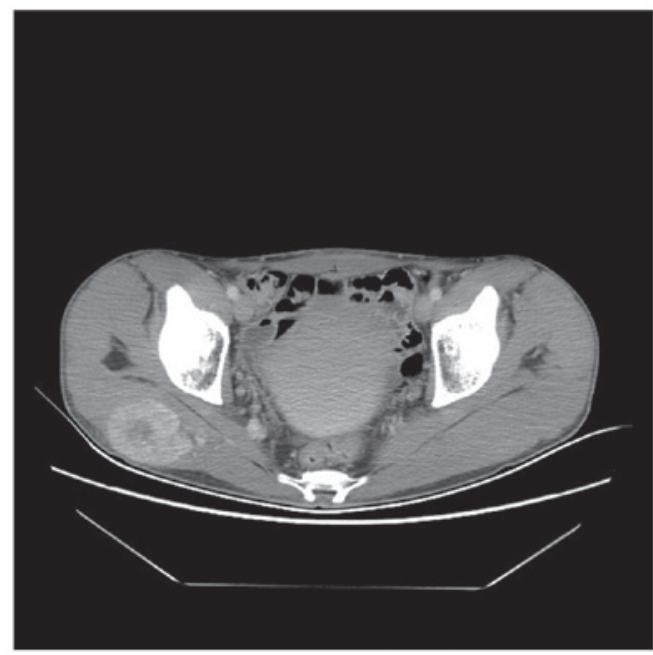

Venous phase

Figure 1. CT scan showing a low density mass in the right ectogluteus. There was strong enhancement in the arterial phase of the enhanced scan and the strengthening degree reduced in the venous phase.

on T1-weighted imaging (WI) and hyperintensity on T2WI. Cystic degeneration and necrosis were found in 2 cases. The solid component of the tumors showed marked enhancement on contrast-enhanced MRI (Fig. 2). Microscopic analysis

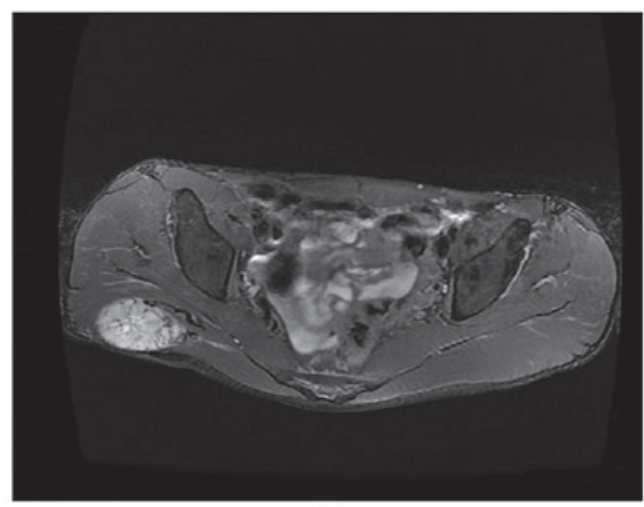

T2

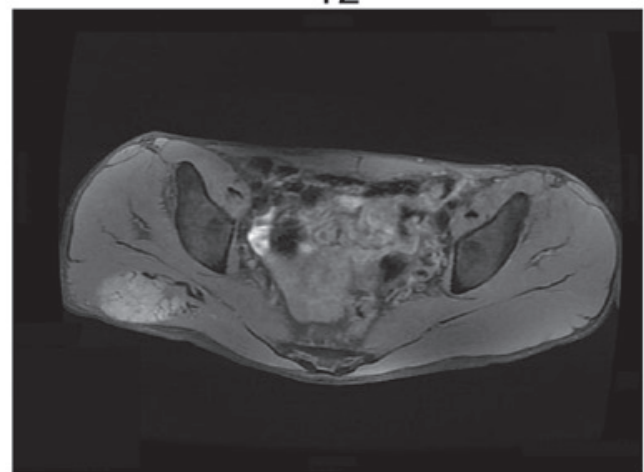

T1 Fat-sat

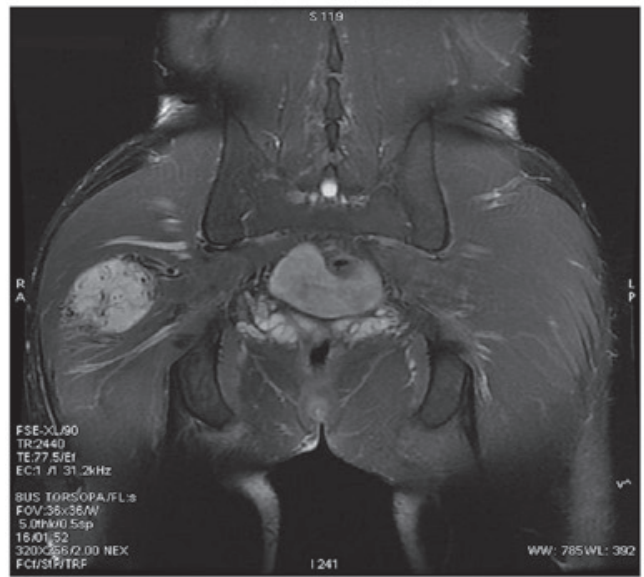

T2 COR

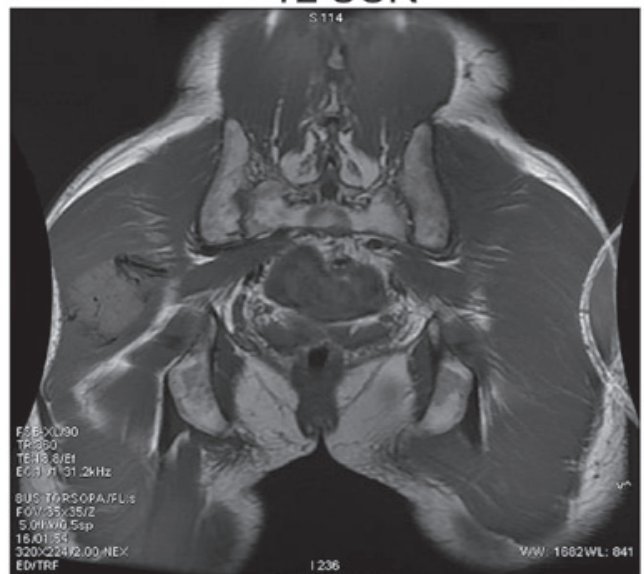

T1 COR

Figure 2. Plain magnetic resonance imaging (MRI) scan showing the equal T1 and long T2 signals, the clear boundary and high signals on diffusion-weighted imaging (DWI). The signals were not even and the tumor size was $\sim 3.9 \times 4.7 \times 5.3 \mathrm{~cm}$. Contrast-enhanced MRI scan showing the mass with inhomogeneous enhancement. Fat-sat, fat-saturated; Cor, coronal; C, contrast. 


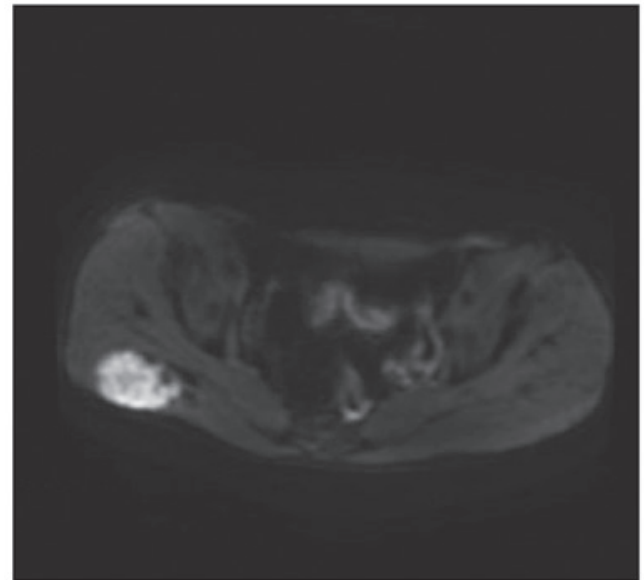

DWI

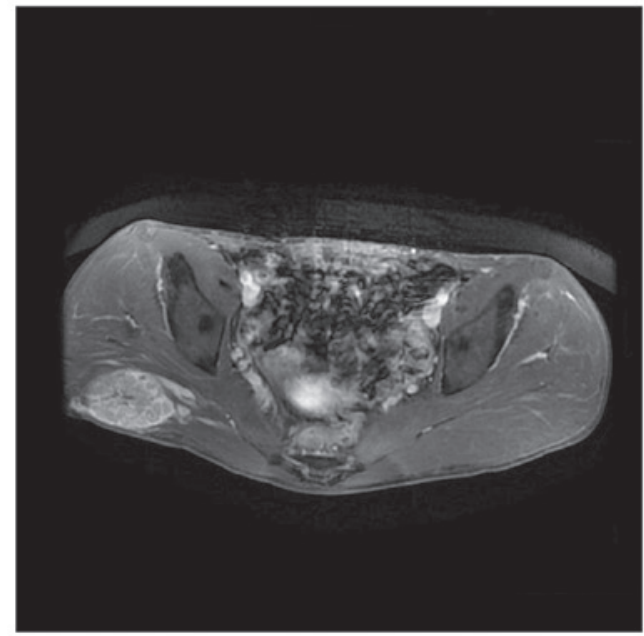

$\mathrm{T} 1+\mathrm{C}$

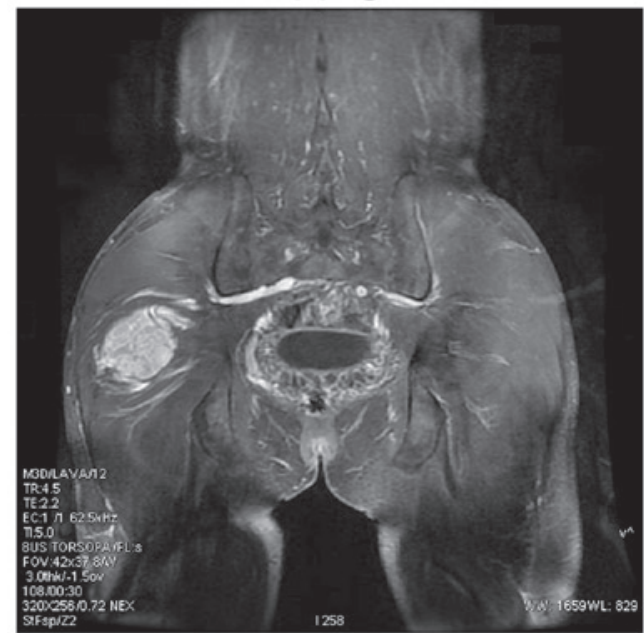

$\mathrm{COR}+\mathrm{C}$

Figure 2 continued. Plain magnetic resonance imaging (MRI) scan showing the equal T1 and long T2 signals, the clear boundary and high signals on diffusion-weighted imaging (DWI). The signals were not even and the tumor size was $\sim 3.9 \times 4.7 \times 5.3 \mathrm{~cm}$. Contrast-enhanced MRI scan showing the mass with inhomogeneous enhancement. Cor, coronal.

revealed tumor cells with granular cytoplasm arranged in alveolar or solid structures separated by sinusoidal vessels. Crystals were present in the cytoplasm of the tumor cells following periodic acid-Schiff (PAS) staining. Immunohistochemically, 4 cases were positive for MyoD1, 1 was positive

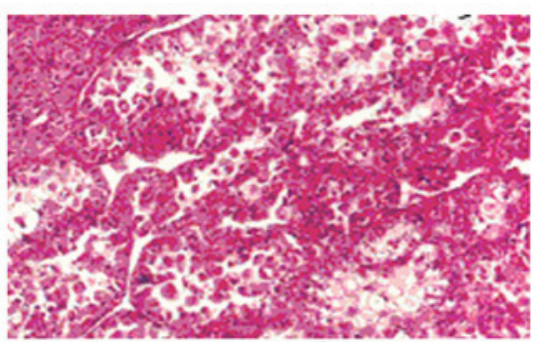

low-power field

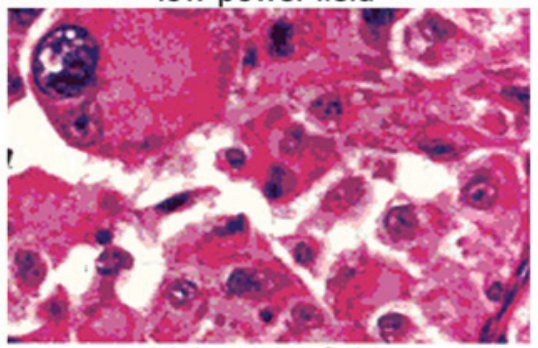

high-power field

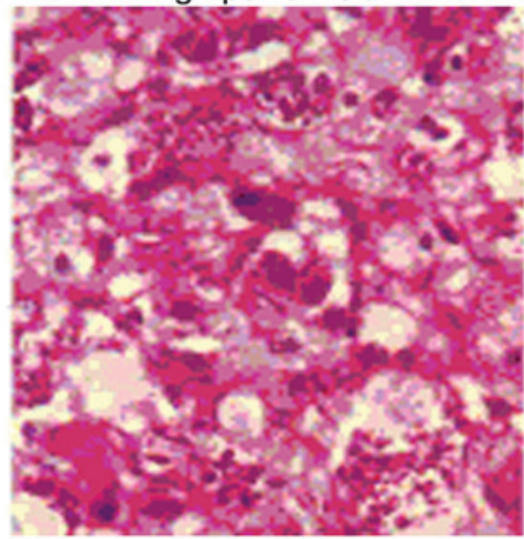

PAS staining

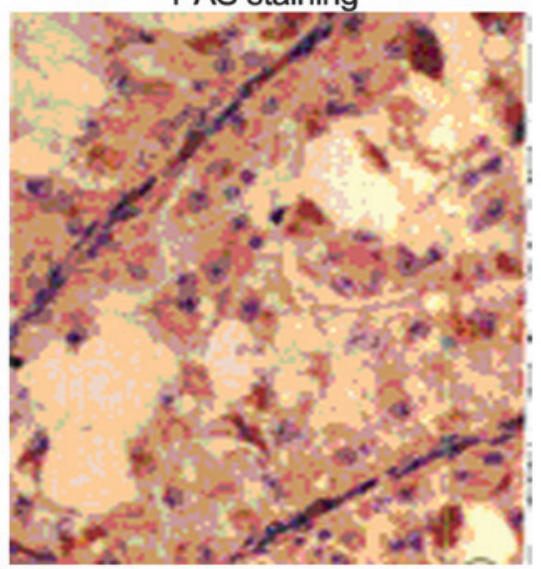

immunohistochemical staining

Figure 3. In low-power field, the tumor cells were arranged alveolar, abundant sinusoidal capillaries could be seen between the gland alveolus. In high-power field, the cytoplasm of tumor cell was rich, including eosinophil granule, clear entoblast. A few cells had double or multi nucleolus. After PAS staining, the club-shaped or spiculate crystallinesubstance could be seen in the cytoplasm. After immunohistochemical staining (Envision methods), brown kernels could be seen in the cytoplasm, MyoD1 positive.

for desmin, 2 were positive for S-100, 5 were positive for neuron-specific enolase and 5 were positive for vimentin. All ASPS cases were negative for AE1/AE3, cytokeratin, epithelial membrane antigen, smooth muscle actin, muscle-specific actin and synaptophysin (Fig. 3). 


\section{Discussion}

Upon pathological examination, ASPS always has an incomplete capsule and the edge is nodular. When the tumor becomes large, it can cause hemorrhagic necrosis, with tumor cells arranged loosely in substantial aciniform or nested structures. Central cell nests can appear with degeneration or necrosis, the cytoplasm is positive for PAS staining, and the cells contain rod- or bar-shaped crystals, all of which contribute to the tumor diagnosis (5). The blood supply of the tumor tissue is rich; the presence of bulky, twisted blood vessels around the tumor and a large amount of bleeding can be observed during surgery (6).

The imaging results, particularly on MRI, can reflect the clinical and pathological characteristics of ASPS to a certain extent. As patients are often experience a long course of disease and the clinical symptoms are not pronounced, the majority of patients present to hospital with a larger tumor or serious symptoms prior to treatment. In the present study, the mean tumor volume was $\sim 4.1 \times 4.8 \times 3.3 \mathrm{~cm}$, which is consistent with the tumor sizes of ASPS. Upon pathological examination, the tumors had a compact nature and were elliptical in shape. MRI exhibited moderate $\mathrm{T} 1$ and long $\mathrm{T} 2$ signals for the soft-tissue mass. Upon growth of the tumor, hemorrhage and necrosis can be caused, and in certain cases in the present study, the central region exhibited necrosis or/and cystic change. The shape of the cystic region was irregular, showing markedly abnormal long T1 and T2 signals. The blood supply of the tumor was rich, so it exhibited strong enhancement.

The MRI of the tumor boundary reflects its invasive ability $(6,7)$, and a previous study (8) has reported that the tumor peripheral nodule formation is due to the breakthrough of sarcoma cells through the cell membrane and into the 'tumor response area'. In the present study, 2 tumors exhibited no clear boundary between the local and surrounding tissue, showing continuity. There were soft-tissue nodules around the tumors in 4 cases, which meant that the tumor cells grew to break through the thin film, indicating a malignant tumor. Another study (9) reported that the transfer of ASPS metastases mainly occurred via the blood, and that the lung was the most common metastatic site, being affected in $42-65 \%$ of all patients, while lymph node metastasis occurred in only $10 \%$ of the patients (10).

For the differential diagnosis, ASPS must be distinguished from rhabdomyosarcoma, fibrosarcoma, malignant fibrous histiocytoma, synovial sarcoma and atypical lipoma. Rhabdomyosarcoma is large and lobulated, with moderate $\mathrm{T} 1$ and long T2 or mixed signals. Tumor necrosis in common, with enhanced scans showed marked enhancement. Pathological diagnosis may also aid the differentiation. With regard to fibrosarcoma, T1WI demonstrates equal or slightly low signals, and T2WI demonstrates uneven slightly high signal within the tumor. Hemorrhage and necrosis can occur, and the tumor easily invades neighboring bone. Malignant fibrous histiocytoma consists of lobulated soft-tissue masses, with moderate $\mathrm{T} 1$ signals and heterogeneous high signals on T2WI. The tumor can present as a bleeding cyst, without enhancement on T1W1. When the fiber content in the tumor is large, an irregular low signal is visible on T2WI, which can be used for identification purposes. Synovial sarcoma is a nodular or lobulated soft-tissue mass. The tumor commonly exhibits focal calcification, with moderate $\mathrm{T} 1$ and long $\mathrm{T} 2$ signals due to hemorrhage, necrosis and calcification. The tumor signal is often uneven (11). With regard to atypical lipoma, the fat content of well-differentiated liposarcoma is rich and easy to identify. However, less-differentiated liposarcoma, particularly pleomorphic liposarcoma, contains a large quantity of immature adipose tissue, with a soft-tissue signal, which requires careful identification (12).

Overall, ASPS is a rare, malignant, soft-tissue tumor, and although the incidence rate is low, the clinical and imaging diagnosis should not be overlooked. For young and middle-aged patients, the possibility of ASPS should be considered for slow-growing tumors that occur in the deep muscles. The characteristics of ASPS upon imaging are mainly the presence of a large, lobulated mass with an incomplete capsule and nodules of soft tissue around the tumor. The tumor can exhibit necrosis or metastasize when it becomes larger. On MRI, the tumor parenchyma show moderate $\mathrm{T} 1$ and long $\mathrm{T} 2$ signals, with marked enhancement on the contrast scans. Although these signs are non-specific and the diagnosis still relies on the pathology, the MRI manifestations can reflect the characteristics of malignant tumors to a certain extent, enabling a qualitative diagnosis and guiding clinical pre-operative treatment.

\section{References}

1. Enzinger FM and Weiss SW: Alveolar soft part sarcoma. In: Soft tissue tumors. 4th edition. Mosby Press, St. Louis, pp1509-1521, 2002.

2. Ladanyi M, Lui MY, Antonescu CR, Krause-Boehm A, Meindl A, Argani P, Healey JH, Ueda T, Yoshikawa H, Meloni-Ehrig A, et al: The $\operatorname{der}(17) \mathrm{t}(\mathrm{x} ; 17)(\mathrm{p} 11 ; \mathrm{q} 25)$ of human alveolar soft part sarcoma fuses the TFE3 transcription factor gene to ASPL, a novel gene at 17q25. Oncogene 20: 48-57, 2001.

3. Marchac A, Picard A, Landman-Parker J, Larroquet M, Vazquez MP and Franchi G: A pediatric case of alveolar soft part sarcoma. Rev Stomatol Chir Maxillofac 108: 547-550, 2007 (In French).

4. Folpe AL and Deyrup AT: Alveolar Soft-part Sarcoma: A review and update. J Clin Pathol 59: 1127-1132, 2006.

5. Akiyama Y, Baba T, Ibayashi Y, Asai Y and Houkin K: Alveolar soft part sarcoma in brain with cardiac metastasis: A case report. Int J Cardiol 114: e93-e95, 2007.

6. Nakano H, Tateishi A, Imamura T, Miki H, Ohno T, Moue T, Sekiguchi M, Unno K, Abe S, Matsushita T, et al: RT2PCR suggests human skeletal muscle origin of alveolar soft part sarcoma. Oncology 58: 319-323, 2000.

7. Moulton JS, Blebea JS, Dunco DM, Braley SE, Bisset GS III and Emery KH: MR imaging of soft tissue masses: diagnostic efficacy and value of distinguishing between benign and malignant lesions. AJR Am J Roentgenol 164: 1191-1199, 1995.

8. Saito T, Oda Y, Kawaguchi K, Takahira T, Yamamoto H, Sakamoto A, Tamiya S, Iwamoto Y and Tsuneyoshi M: Possible association between tumor-suppressor gene mutations and hMSH2 /hMLH1 inactivation in alveolar soft part sarcoma. Hum Pathol 34: 841-849, 2003.

9. Wang CK, Li CW, Hsieh TJ, Chien SH, Liu GC and Tsai KB: Characterization of bone and soft-tissue tumors with in vivo $1 \mathrm{~h}$ mr spectroscopy: Initial results. Radiology 232: 599-605, 2004.

10. Negendank WG, Sauter R, Brown TR, Evelhoch JL, Falini A, Gotsis ED, Heerschap A, Kamada K, Lee BC and Mengeot MM: Proton magnetic resonance spectroscopy in patients with glial tumors: A multicenter study. J Neurosurg 84: 449-458, 1996.

11. Lorigan JG, O'Keeffe FN, Evans HL and Wallace S: The radiologic manifestations of alveolar soft-part sarcoma. AJR Am J Roentgenol 153: 335-339, 1989.

12. Aiken AH and Stone JA: Alveolar soft-part sarcoma of the tongue. AJNR Am J Neuroradiol 24: 1156-1158, 2003. 